\title{
Eighty cases of conservative treatment of ectopic pregnancy with combined use of traditional Chinese medicine and Western medicine.
}

\author{
Fengde Jing ${ }^{1 \#}$, Yanchun Wang ${ }^{2 \#}$, Peng Qiao ${ }^{1}$, Yubin Liu ${ }^{3 *}$ \\ ${ }^{1}$ Department of Traditional Chinse Medicine, Yantaishan Hospital, Yantai, PR China \\ ${ }^{2}$ Department of Obstetrics and Gynecology, Yantai Hospital of Traditional Chinese Medicine, Yantai, PR China \\ ${ }^{3}$ Department of Rehabilitation, Yantaishan Hospital, Yantai, PR China \\ \#Contributed equally
}

\begin{abstract}
Objective: To observe the clinical effect of conservative treatment of ectopic pregnancy with the combination of methotrexate+mifepristone+traditional Chinese medicine.

Methods: Eighty patients diagnosed with ectopic pregnancy with conservative treatment indication and/or requirements were treated with methotrexate+mifepristone+traditional Chinese medicine.

Results: sixty-four patients' serum $\beta$-HCG values dropped to the normal level. Among them fifty-eight patients' serum $\beta$-HCG values dropped to normal level with the mass completely disappearing. Ten patients had laparoscopic surgery intervention in the process of conservative treatment. Three patients were intra-operatively diagnosed with ruptured ectopic pregnancy, and six were intra-operatively diagnosed with tubal pregnancies, including one accompanied by pelvic adhesions. One patient was intra-operatively diagnosed with cornual pregnancy with abdominal bleeding.

Conclusion: The conservative treatment of ectopic pregnancy using the combination of Methotrexate +Mifepristone+traditional Chinese medicine. This method is reliable, simple, convenient, with low cost and fewer side effects, and easy to be accepted by the majority of patients, thus being worth promoting in clinical treatment.
\end{abstract}

Keywords: Ectopic pregnancy, Chinese medicine, Western medicine, Methotrexate, Mifepristone.

Accepted on May 26, 2017

\section{Introduction}

Chinese medicines have become very popular and are widely applied to different kinds of medical conditions during pregnancy. It promotes both mothers' and fetuses' health, relieves and cures common disorders in women. It has been used as a main stream medicine in China with a longer history than Western medicines [1].

Ectopic pregnancy, which occurs in one out of every 100 cases [2], is the most commonly acute abdomen in gynecology. Recent years have witnessed the rising incidence of ectopic pregnancy at home and abroad, with significant rise among the unmarried and the nulliparas. Due to the advance of diagnosis and treatment technologies, most of the ectopic pregnancies can be diagnosed in the early stage before rupture. Although the efficacy of laparoscopic salpingectomy is reliable, it brings great pain and life-long regret to patients with only one fallopian tube left.

More and more ectopic pregnancy patients got early diagnosed due to the improving and application of vaginal ultrasound, diagnostic curettage and Beta-Human Chorionic Gonadotropin
( $\beta$-HCG) detection. This provided opportunities for conservative treatment with medicines. Conservative treatment is not only beneficial for keeping tubal function, but also avoiding complications after surgeries, which may be helpful to the recovery of the patients. In recent years, conservation treatment was widely used for ectopic pregnancy in China. The efficacy of methotrexate in combination with mifepristone was evaluated in clinical practice. This combination induces the death and absorption of the ectopic pregnancy embryo tissue. It is thus regarded as a quite safe, effective and less adverse reactions method [3].

Nevertheless, given the conflicting information in the public domain regarding the perceived safety and potential dangers of acupuncture during pregnancy, there is understandably an undertone of anxiety regarding its use during the antenatal period. Arguably the biggest fear among practitioners is the scenario in which a patient experiences a miscarriage or delivers prematurely and the acupuncture treatment is blamed by association. Professional anxiety surrounding the treatment of pregnant women is not specific to acupuncture. Ultimately, however, it must be remembered that there is an expected rate 
of naturally-occurring complications within the pregnant population, irrespective of any intervention; therefore, practitioners need to be familiar with the background incidence of adverse outcomes to assess whether the observed frequency of such complications matches that which is expected, whether it be in the acupuncture arm of a Randomised Controlled Trial (RCT) or an audit within clinical practice [4].

Therefore, the therapy that can retain the patients' reproductive function is very important. From January 2012 to February 2015, our hospital treated eighty patients with ectopic pregnancy adopting conservative therapy using a combination of methotrexate+mifepristone+traditional Chinese medicine, the effect of which is satisfactory.

\section{Methodology}

\section{General information}

A total of 80 patients with unruptured ectopic pregnancy who received treatment during the period from January 2012 to February 2015 were selected as the research objects. These patients meet the diagnostic criteria specified in the chapter of ectopic pregnancy of the seventh edition of the text book Obstetrics and Gynecology, as well as all the following requirements of conservative therapy: (1) stable vital signs, no active intra-abdominal hemorrhage and pelvic fluid $\leq 4 \mathrm{~cm}$ as shown in B-scan image; (2) no significant abdominal pain; (3) B-scan image shows the maximum diameter of ectopic pregnancy mass $\leq 6 \mathrm{~cm}$ or the maximum diameter of ectopic pregnancy mass $>6 \mathrm{~cm}$, but the patients refused surgery, required conservative treatment and signed the Informed Consent; (4) patients' serum $\beta$-HCG level $\leq 5000 \mathrm{U} / \mathrm{L}$ or $\beta$ HCG level $>5000 \mathrm{U} / \mathrm{L}$, but patients refused surgical treatment, required conservative treatment and signed the Informed Consent; (5) normal liver and kidney function and results of routine blood test; (6) patients required conservative treatment with medication and signed for it. Patients of the group aged from 19 to 40 with menopause up to $30 \sim 81$ days and $\beta$-HCG level at 45.93 18181 U/L. Among them, the $\beta$-HCG level was at $45.93 \sim 600 \mathrm{U} / \mathrm{L}$ in 29 cases, $601 \sim 1000 \mathrm{U} / \mathrm{L}$ in 11 cases, $1001 \sim 2000 \mathrm{U} / \mathrm{L}$ in 14 cases, $>6000 \mathrm{U} / \mathrm{L}$ in 6 cases (including $\beta$ HCG level $>10000 \mathrm{U} / \mathrm{L}$ in 3 cases). As for the size of the mass, the biggest was $66 \mathrm{~mm} \times 72 \mathrm{~mm} \times 52 \mathrm{~mm}$ and the smallest 10 $\mathrm{mm} \times 7 \mathrm{~mm} \times 12 \mathrm{~mm}$.

\section{Therapeutic approach}

Daily $20 \mathrm{mg}$ of methotrexate was intramuscularly injected for 5 consecutive days; $50 \mathrm{mg}$ of mifepristone was taken orally twice a day for 5 consecutive days. Simultaneously, a dose of self-made ectopic pregnancy soup, which was made of sparganii rhizoma 20 40 g, curcuma zedoary $20 \sim 40$ g, selfheal 20-40 g, spina gleditsiae 12 24 g, turtle shell 20 40 g, stiff silkworm $20 \mathrm{~g}$, scolopendra $1 \sim 3 \mathrm{~g}$, radices trichosanthis $20 \sim 40$ $\mathrm{g}$, notoginseng powder $6 \sim 12 \mathrm{~g}$, typhae pollen $10 \sim 20 \mathrm{~g}$ and radix scrophulariae $15 \mathrm{~g}$, were taken orally each day in the morning and evening respectively for five consecutive days. All the above medicines should be taken after being mixed with hot water and do not require decocting. After taking the dosage for five days, the patients were examined with B-scan to check the mass (patients with increased abdominal pain and rising HCG level during the mediation were excluded). Patients with the diameter of the mass $\leq 4 \mathrm{~mm}$ and serum $\beta$ $\mathrm{HCG} \leq 2000 \mathrm{U} / \mathrm{L}$ were given lower dose of the above medication; patients with the diameter of the mass $>4 \mathrm{~mm}$ and serum $\beta$-HCG $>2000 \mathrm{U} / \mathrm{L}$ were given higher dose of the above medication. For patients with irregular vaginal bleeding, charred node of the lotus rhizome and charred human hair were added; for patients with abdominal pain, corydalis rhizoma, radix paeoniae alba and trogopterus dung were added and for patients with nausea, pinellia ternate, inula flower, etc. were added. After taking the medication, blood pressure, pulse, abdominal pain and vaginal bleeding were closely observed. On day 4 and 7 after treatment, serum $\beta-H C G$ was reexamined. Patients with $\beta$-HCG value drop less than $25 \%$ on day 7 were given the same western medicine. Afterwards, the patients had B-scan and serum $\beta$-HCG examination every week until the mass of ectopic pregnancy $\leq 2 \mathrm{~mm}$ or disappeared and serum $\beta$-HCG level lowered to normal level (about half of these patients failed to follow the doctor's advice to continue the treatment with traditional Chinese medicine).

\section{Observation indexes}

The mass of ectopic pregnancy, serum $\beta$-HCG level, the change of the abdominal pain, liver function.

\section{Efficacy determination}

Cure: clinical symptoms disappeared, no abdominal pain, and serum $\beta$-HCG level down to normal or close to normal. B-scan image shows that the mass of ectopic pregnancy is no larger than $2 \mathrm{~mm}$ or was completely reabsorbed by the body; Significant efficacy: (1) $\beta$-HCG dropped to normal or close to normal. But the patients had poor compliance and failed to follow the doctor's advice to keep regular outpatient review. So the last time they had outpatient review, the maximum diameter of the pelvic mass was larger than $2 \mathrm{~mm}$. (2) After the treatment with combined use of traditional Chinese medicine and Western medicine, patients' $\beta$-HCG dropped by $62 \%$ or more; pelvic mass became smaller or insignificantly increased and had no abdominal pain or other discomfort. Hence, patients signed for discharge from the hospital, and didn't have outpatient review after the discharge. Failure: aggravated clinical symptoms, increased vaginal bleeding or abdominal hemorrhage, severe abdominal pain, increase or unsatisfactory decrease of serum $\beta$-HCG value; B-scan image shows expanded mass, thus the patients had operative treatment.

\section{Results}

\section{The change of $\beta-H C G$ levels}

Of the 80 cases, the $\beta$-HCG levels of 2 cases increased in the process of treatment, the $\beta$-HCG levels of other 78 cases were markedly reduced. The shortest time for serum $\beta$-HCG value to decline to the normal was 6 days, while the longest up to 2 
months, with an average of 23.31 days. It took an average of 22.34 days for the cured 58 patients to lower serum $\beta$-HCG to normal level and in significantly effective in the $\beta$-HCG levels dropped to normal average with 26.123 days in 20 patients, which operative treatment effective significantly.

\section{The mass of ectopic pregnancy}

Of the 80 cases, 9 cases of ectopic pregnancy patients carried ultrasound examination of gynecopathy, the mass of ectopic pregnancy markedly augmentation and the mass of ectopic pregnancy of other patients with significantly reduced or disappeared

\section{The change of the abdominal pain}

Of the 80 cases, 10 patients suffering from aggravated abdominal pain, other patients with abdominal pain disappeared.

\section{Treatment effects}

Of the 80 cases, 58 cases met the cure standard, accounting for $72.5 \% ; 12$ cases showed significant efficacy, accounting for $15 \%$, thus the total effective rate was $87.5 \%$. 10 cases of failure account for $12.5 \%$. In the course of treatment, 10 patients who received surgery suffering from intolerable sudden abdominal pain, in addition to abdominal tenderness and rebound tenderness $(+)$, and the mass of ectopic pregnancy markedly augmented with ultrasound examination of gynecopathy, which were intraoperatively diagnosed with ruptured ectopic pregnancy. Three patients were intraoperatively diagnosed with ruptured ectopic pregnancy. Six were intraoperatively diagnosed with tubal pregnancy. One patient needed surgery intervention due to increased abdominal pain, in addition to rebound tenderness (-), and the mass of ectopic pregnancy have no augmentation with ultrasound examination of gynecopathy and was intraoperatively diagnosed with cornual pregnancy with abdominal bleeding. Two patient need surgery intervention because of the $\beta$-HCG levels with significant higher, marked augmentation of the mass of ectopic pregnancy, abdominal tenderness $(+)$ and rebound tenderness (-), and were intraoperatively diagnosed with tubal pregnancy, including one accompanied by pelvic adhesions.

Among these 10 postoperative patients, before the conservative treatment, there were two cases with the serum $\beta$-HCG value $<600 \mathrm{U} / \mathrm{L}$; two cases with the serum $\beta$-HCG value between 1000 2000 U/L; three cases with the serum $\beta$-HCG value between $3000 \sim 4000 \mathrm{U} / \mathrm{L}$; one case with the serum $\beta$ HCG value between 4000 5000 U/L; two cases with the serum $\beta$-HCG value higher than $10000 \mathrm{U} / \mathrm{L}$.

\section{Discussion}

With the increasingly frequent application of caesarean, surgical abortion and intrauterine devices in recent years, the incidence of ectopic pregnancy is increasing year by year and shows the trend of occurrence at earlier age. The more knowledge about ectopic pregnancy and the improvement of diagnostic technologies make the diagnosis of majority of the ectopic pregnancies before the rupture possible. Laparoscopic surgery leaves fewer traumas compared with traditional laparotomy, showing reliable efficacy. However, it's still an invasive surgery, which not only increases the probability of pelvic adhesions and infertility in the future, but also increases psychological burden for the unmarried. Conservative treatment with drugs alleviates patients' pain, meets their psychological needs, and relieves the financial burden for patients. The key to the successful cure of ectopic pregnancy is to kill the survived embryo and trophoblast cells. Mifepristone has the function of anti-progesterone and anti-glucocorticoid, blocks the function of progesterone via the competing with progesterone receptor and antagonizing progesterone activity, and finally kills the embryo and promotes its discharge [5]. Methotrexate is a folic acid antagonist which can inhibit dihydrofolate reductase, interfere with protein synthesis of DNA and RNA, and restrain the division of embryonic trophoblast cell, thereby resulting in embryonic atrophy and death. Methotrexate is extensively used in killing embryos with good efficacy [6]. Methotrexate produces severe side effects in clinical application. Gastrointestinal reaction, oral ulcer, bone marrow suppression, liver and kidney damage and other adverse reaction are commonly observed. The combination of mifepristone and Methotrexate shows synergistic effect and decreases the drug dose at the same time, thus reduces side effects and ensures the efficacy [7].

Ectopic pregnancy falls into the category of "abdominal mass" in traditional Chinese medicine, which is mostly caused by qi stagnation and blood stasis. Traditional Chinese medicine holds that ectopic pregnancy is a disease featuring blood stasis in lower abdomen. The embryo is located in the wrong place, which blocks qi circulation. The stagnation of qi causes blood stasis and damage to collaterals. Since the blood fails to run through meridians, it spills out. Therefore, the main principle of the treatment is to promote blood circulation, remove blood stasis and resolve lumps and nodes along with feticide. The self-made ectopic pregnancy soup was made mainly of sparganii rhizoma, curcuma zedoary, selfheal, spina gleditsiae, turtle shell, stiff silkworm, scolopendra, radices trichosanthis, notoginseng powder, typhae pollen and radix scrophulariae. Sparganii rhizoma and curcuma zedoary can remove blood stasis, soften and resolve hard mass and promote qi circulation and kill pain; selfheal, turtle shell, stiff silkworm, scolopendra and radix scrophulariae can soften and resolve hard mass, dispel phlegm and resolve nodes; spina gleditsiae can eliminate phlegm (inside the thoracic diaphragm), promote blood circulation and remove meridian obstruction; notoginseng powder can resolve blood stasis, promote blood circulation and stop bleeding without leaving stasis, thus removing blood stasis and promoting tissue regeneration; typhae pollen can induce astringency, stop bleeding without leaving stasis, promote blood circulation and remove blood stasis. The combination of typhae pollen and notoginseng powder can strengthen the sparganii rhizoma and curcuma zedoary'sfunction of removing blood stasis, and prevent bleeding due to excessive power of blood circulation 
activation. Radices trichosanthis can clear heat and promote salivation, subside the swelling and prevent early pregnancy. The studies of modern clinical Chinese pharmacology have shown that: sparganii rhizome, curcuma zedoary, pollen typhae, stiff silkworm and spina gleditsiae can prevent early pregnancy caused embryo necrosis, absorption and discharge, soften and strip the embryo, and has the antibacterial, antiinflammatory, antithrombotic and analgesic effect. The combined use of drugs can activate blood circulation and remove stasis, kill embryos, remove masses and nodes and activate meridians. Moreover, it can also relax blood vessels, improve microcirculation, promote dissipation of blood stasis, eliminate pelvic inflammation, enhance the function of the lymphatic system, reduce the synthesis of collagenous fiber, promote collagenase and fibrinolysin activity, enhance fibrinolytic system, soften the organization and promote tissue repair and regeneration. The traditional Chinese medicines mentioned above can also clear the fallopian tube, restore oviduct function, improve the success rate of the next normal pregnancy, and reduce the recurrence of ectopic pregnancy [8].

Of the ten patients who failed in conservative treatment in the group, there were two cases with the serum $\beta$-HCG value $<600$ $\mathrm{U} / \mathrm{L}$ before the conservative treatment, accounting for $6.89 \%$ of the total number of cases with serum $\beta-\mathrm{HCG}<600 \mathrm{U} / \mathrm{L}$ before the conservative treatment; two cases with the serum $\beta$-HCG value between $1000 \sim 2000 \mathrm{U} / \mathrm{L}$ before the conservative treatment, accounting for $14.28 \%$ of the total number of cases with serum $\beta$-HCG between 1000 2000 U/L before conservative treatment; three cases with the serum $\beta$-HCG value between $3000 \sim 4000 \mathrm{U} / \mathrm{L}$ before the conservative treatment; accounting for $50 \%$ of the total number of cases with serum $\beta-\mathrm{HCG}$ between 3000 4000 U/L before the conservative treatment; one case with the serum $\beta$-HCG value between $4000 \sim 5000 \mathrm{U} / \mathrm{L}$ before the conservative treatment, accounting for $50 \%$ of the total number of cases with serum $\beta$ HCG between $4000 \sim 5000 \mathrm{U} / \mathrm{L}$ before the conservative treatment; two cases with the serum $\beta$-HCG value higher than $10000 \mathrm{U} / \mathrm{L}$ before the conservative treatment, accounting for $66.67 \%$ of the total number of cases with serum $\beta$-HCG higher than $10000 \mathrm{U} / \mathrm{L}$ before the conservative treatment. The above data shows that the success rate of conservative treatment is associated with serum $\beta$-HCG value. Namely, the higher the $\beta$ HCG value is, the lower the success rate of conservative treatment will be. Certainly, the efficacy is also closely correlated with the size of ectopic pregnancy mass before the treatment. Therefore, given a certain risk of conservative treatment for ectopic pregnancy, it should be carried out when the conditions for surgery are met. Conservative treatment of ectopic pregnancy with combined use of Methotrexate
+ mifepristone+traditional Chinese medicine not only displays the respective effects of these drugs, but plays a complementary role in that the use of methotrexate and mifepristone can overcome the weakness of power shortage of traditional Chinese medicine in killing embryos, while the traditional Chinese medicine can bring its advantages of stimulating blood circulation and removing stasis and nodes into play. This method is reliable, simple, convenient, with low cost and fewer side effects, and easy to be accepted by the majority of patients, thus being worth promoting in clinical treatment.

\section{References}

1. Li L, Han LS, Jiang XL, Leung PC, Wang CC. Overview of Contraindicated Chinese Medicines for Pregnancy. In Chinese Medical Therapies for Diabetes, Infertility, Silicosis and the Theoretical Basis. InTech, 2017.

2. Jie L. Obstetrics and Gynecology (Edn 6), People's Medical Publishing House Beijing 2004.

3. Wan S, Xiang Y, Fang W, Huang D. The effect of methotrexate in combination with mifepristone on ectopic pregnancy: a meta-analysis. Int J Clin Exp Med 2016; 9: 14990-15003.

4. Carr DJ. The safety of obstetric acupuncture: forbidden points revisited. Acupuncture Med 2015.

5. Lanlan F, Meiling H. Clinical observation on persistent ectopic pregnancy prevention and treatment with the combined therapy of mifepristone and methotrexate. China Practical Med 2010; 5: 103.

6. Namoum AB. Medical management of ectopic pregnancy. Clinobstl Cynecol 1998; 41: 382.

7. Ting C. Analysis on 35 cases of conservative treatment of ectopic pregnancy with combined therapy of mifepristone and methotrexate. J China Tradit Chinese Med Inform 2010; 2: 217.

8. Hongying L. Experience of treating ectopic pregnancy with combined use of traditional chinese medicine and western medicine. Mod J Integr Tradit Chinese Western Med 2005; 14: 2586 .

\section{*Correspondence to}

Yubin Liu

Department of Rehabilitation

Yantaishan Hospital

PR China 